\title{
Functional Clustering of Neurons in Motor Cortex Determined by Cellular Resolution Imaging in Awake Behaving Mice
}

\author{
Daniel A. Dombeck, ${ }^{1,3}$ Michael S. Graziano, ${ }^{2,3}$ and David W. Tank ${ }^{1,3}$ \\ Departments of ${ }^{1}$ Molecular Biology and ${ }^{2}$ Psychology, and ${ }^{3}$ Princeton Neuroscience Institute, Princeton University, Princeton, New Jersey 08544
}

\begin{abstract}
Macroscopic (millimeter scale) functional clustering is a hallmark characteristic of motor cortex spatial organization in awake behaving mammals; however, almost no information is known about the functional micro-organization ( $\sim 100 \mu \mathrm{m}$ scale). Here, we optically recorded intracellular calcium transients of layer $2 / 3$ neurons with cellular resolution over $\sim 200-\mu \mathrm{m}$-diameter fields in the forelimb motor cortex of mobile, head-restrained mice during two distinct movements (running and grooming). We showed that the temporal correlation between neurons was statistically larger the closer the neurons were to each other. We further explored this correlation by using two separate methods to spatially segment the neurons within each imaging field: K-means clustering and correlations between single neuron activity and mouse movements. The two methods segmented the neurons similarly and led to the conclusion that the origin of the inverse relationship between correlation and distance seen statistically was twofold: clusters of highly temporally correlated neurons were often spatially distinct from one another, and (even when the clusters were spatially intermingled) within the clusters, the more correlated the neurons were to each other, the shorter the distance between them. Our results represent a direct observation of functional clustering within the microcircuitry of the awake mouse motor cortex.
\end{abstract}

\section{Introduction}

The principle of wiring economy asserts that connected neurons should be physically near to each other to reduce the overall length of the wire connections. The effect of minimizing the costs associated with wiring up distant neurons is an evolutionary advantage conferred upon the organism. This idea of wiring economy dates back to Cajal (1899) and has since been used to understand the possible origins of topographic retinal mapping onto the visual cortex (Cowey, 1979), the fractional volume of cortical gray matter taken up by axons and dendrites (Chklovskii et al., 2002), the positioning of cortical areas in the primate prefrontal cortex (Klyachko and Stevens, 2003), and the physical placement of ganglia in the nematode nervous system (Cherniak, 1994). One extrapolation of the wiring minimization principle is the idea that "like attracts like": neurons involved in like computations are more likely to be synaptically connected to each other than to other neurons and therefore should be as physically near each other as possible (Mitchison, 1992).

When applied to the mammalian motor cortex, this "like attracts like" idea has helped to explain the functional organization that exists on the macroscopic scale (Aflalo and Graziano, 2006;

Received June 22, 2009; revised Aug. 24, 2009; accepted Sept. 15, 2009.

This work was supported by Princeton University, the National Institutes of Health (Grant 1R01MH083686-01), and The Patterson Trust. We thank F. Collman, G. Stephens, and I. Ozden for helpful discussions and C. Harvey for comments on this manuscript.

Correspondence should be addressed to either Daniel A. Dombeck or David W. Tank, 270C Carl Icahn Laboratory, Washington Road, Princeton University, Princeton, NJ 08544. E-mail: ddombeck@princeton.edu or dwtank@ princeton.edu.

DOI:10.1523/JNEUROSCI.2985-09.2009

Copyright $\odot 2009$ Society for Neuroscience $\quad$ 0270-6474/09/2913751-10\$15.00/0
Graziano and Aflalo, 2007). Different studies have found functional clustering in the form of subregions that emphasize control of specific muscles (Asanuma and Rosén, 1972), direction of hand movement (Georgopoulos et al., 1982, 2007; Amirikian and Georgopoulos, 2003) or ethologically relevant movements (Graziano et al., 2002). These studies, however, used electrode penetration mapping methods (either recording or stimulation) that did not sample multiple sites simultaneously and potentially suffered from cell selection bias. A more direct way to reveal functional clustering would be to use cellular resolution imaging techniques to study the mammalian motor cortex. Furthermore, though the electrode studies demonstrated a macroscopic organization, they only indirectly uncovered information about microscopic clustering that may exist (Amirikian and Georgopoulos, 2003). Imaging methods have described functional organization on the microscopic scale in anesthetized rodents (Kerr et al., 2007; Sato et al., 2007); however, due largely to technical limitations associated with studying the microcircuitry in the cortex of awake mammals, it remains unclear whether any significant functional clustering exists on the microscopic scale. For example, does the same "like attracts like" idea that shapes the macroscopic layout of the motor cortex also apply at the microcircuit level? Using our recently developed methods (Dombeck et al., 2007) to optically image the activity of layer $2 / 3$ neurons within a spatial window of $\sim 200 \mu \mathrm{m}$ in awake mice, we asked whether the forelimb region of the motor cortex contained any spatial clustering in the functional properties of single neurons. The mice were mobile, head-restrained, and performed two distinct movements (running and grooming). We measured the time-varying activity of neurons in the field of view 
and studied whether neurons with similar activity patterns were spatially near each other.

\section{Materials and Methods}

Spherical treadmill and data acquisition. The spherical treadmill and twophoton microscope designs have been previously described (Dombeck et al., 2007). The computer mouse system used to record ball rotation, the airpuff system and the data acquisition system used to record and synchronize ball rotation, airpuff timing, and two-photon image frame timing are also the same as previously described (Dombeck et al., 2007). A CCD camera (DCR-SR200, Sony) recorded the mouse movements. It was synchronized to the two-photon time-series by recording the microscope's slow-galvanometer control voltage in the camera's audio input. This allowed for the CCD camera movies to be synchronized within one frame to the two-photon time-series movies. The camera was used in IR ("Night-shot") mode with an added D950/10 filter (Chroma) to block out the two-photon excitation light. The side of the mouse contralateral to the two-photon imaging hemisphere was illuminated by $945 \mathrm{~nm}$ IR-LEDs (RL5-IR2730, Super Bright LEDs).

Animals, training, and surgery. All experiments were performed in compliance with the Guide for the Care and Use of Laboratory Animals (http://www.nap.edu/readingroom/books/labrats/). Specific protocols were approved by the Princeton University Institutional Animal Care and Use Committee. Imaging experiments were performed on 10 male B6CBAF1/J mice (P36-P44). All of the mice underwent two training sessions in which they ran freely on the floating ball, as described previously (Dombeck et al., 2007). Three of the mice then underwent further training in which they were implanted with a temporary head-plate [affixed to the skull with a small amount of Meta-bond (Parkell)] and were head-restrained on the free-floating ball in complete darkness for $60 \mathrm{~min}$ periods for 3 consecutive days. Honey was periodically applied to the whiskers of these mice (to increase the frequency of grooming, see below) during the head-restrained training sessions. The other 7 mice did not receive this additional head-restrained training and were not headrestrained until the imaging experiments. The point of this training was to test if the main results in this research were affected by habituating the mice to the experimental conditions before the imaging experiments; however, no significant differences were seen (data not shown). Therefore, we report here the combined results from all 10 mice ( 7 non-headrestrained trained and 3 head-restrained trained).

Following the training sessions, the mice were anesthetized with isofluorane, the temporary head-plates on the 3 head-restrained trained mice were removed, and a 3-mm-diameter circular craniotomy (circle centered at 1.75 $\mathrm{mm}$ lateral and $0.13 \mathrm{~mm}$ rostral of bregma) was made over one hemisphere and the skull was thinned $\sim 2 \mathrm{~mm}$ rostral and $\sim 2 \mathrm{~mm}$ caudal of the craniotomy edges to allow for the head-plate to fit flush against the edges of the craniotomy. A thin ring of Kwik-sil (World Precision Instruments) was applied around the edges of the craniotomy and the bottom piece of the headplate (see supplemental Fig. $1 D$ for head-plate design, available at www. jneurosci.org as supplemental material) was pushed onto the uncured Kwiksil ring, with the $3 \mathrm{~mm}$ hole lined up to the $3 \mathrm{~mm}$ craniotomy, until it sat flush against the skull (supplemental Fig. $1 A$, available at www.jneurosci.org as supplemental material). When the Kwik-sil set, it had the effect of temporarily holding the head-plate in place until Meta-bond could be applied and it also formed a water and air-tight seal between the bottom of the head-plate and the skull. Meta-bond was then used to bond the bottom-piece of the head-plate to the skull. Uniform thickness Kwik-Sil plugs ( $3 \mathrm{~mm}$ diameter, $0.65 \mathrm{~mm}$ thickness disks) were molded onto \#1 thickness, 3.5-mm-diameter coverslips (Custom coverslips from Erie Scientific) using a custom made mold. The thickness of the plug was designed to apply slight pressure to the surface of the dura to reduce out of focal plane $(Z)$ brain motion. After dye injection (supplemental Fig. $1 A$, available at www.jneurosci.org as supplemental material) and intracortical microstimulation (ICMS) (supplemental Fig. $1 \mathrm{~B}$, available at www.jneurosci.org as supplemental material), the dura was allowed to dry until tacky, a small drop of uncured Kwik-sil was applied to the bottom of the plug, and the coverslip/ plug combination was inserted into the craniotomy and onto the shelf of the bottom piece of the head plate and held rigidly in place with the top piece (supplemental Fig. $1 C$, available at www.jneurosci.org as supple- mental material). When cured, the small drop of Kwik-sil had the effect of bonding the plug to the dura and reducing in-focal plane $(X-Y)$ brain motion.

Following surgery, the mice were head restrained on the ball in complete darkness. Typically, for the 7 non-head-restrained trained mice, it would take $\sim 10-15 \mathrm{~min}$ for the mouse to learn to balance and then begin to walk or run; the 3 head-restrained trained mice could balance, walk and run immediately. Imaging commenced $\sim 1 \mathrm{~h}$, and ended $\sim 2-4 \mathrm{~h}$, after waking from anesthesia.

Dye loading, ICMS, and two-photon microscopy. We initially performed ICMS (details of parameters below) mapping studies in 5 mice not used for imaging to identify the precise stereotaxic location of the forelimb motor cortex in the B6CBAF1/J mouse strain. ICMS in an $\sim 1.0-$ to $1.5-\mathrm{mm}-$ diameter region centered at $1.75 \mathrm{~mm}$ lateral and $0.13 \mathrm{~mm}$ rostral of bregma reliably evoked contralateral forelimb twitches. This area of the cortex was therefore chosen for the subsequent set of imaging experiments.

Dye bolus loading and ICMS were carried out after the bottom piece of the head-plate was affixed to the skull, but before the coverslip and Kwik-sil plug were implanted. A $10 \mathrm{~mm}$ stock of Calcium Green-1 AM in DMSO + 20\% pluronic was diluted tenfold into (in mM): $150 \mathrm{NaCl}, 2.5 \mathrm{KCl}, 10$ HEPES, $\sim 0.05$ SR101 ( $\mathrm{pH}=7.4)$. Filled pipettes (borosilicate, $1.2 \mathrm{~mm}$ OD, $0.6 \mathrm{~mm}$ ID, FHC Co.; P-2000 puller, Sutter Instruments) were beveled (BV-10, Sutter) to facilitate penetration of the intact dura (post-beveling pipette resistance 5-9 $\mathrm{M} \Omega$ ). Pipettes were advanced (MP285, Sutter) through the craniotomy at $39^{\circ}$ with respect to the brain surface and dye injection (1.5-2.0 psi, $\sim 8 \mathrm{~min})$ was made at a depth of $\sim 250 \mu \mathrm{m}$ below the cortical surface (supplemental Fig. $1 \mathrm{~A}$, available at www.jneurosci.org as supplemental material). 2-4 dye injections per mouse were stereotaxically targeted to the forelimb motor cortex, but the exact stereotaxic location varied from mouse to mouse to avoid large blood vessels. Neurons and astrocytes were labeled with the green calcium-sensitive dye, but only the astrocytes were labeled with the red dye SR101 (Nimmerjahn et al., 2004) (Fig. 1Ai). The red SR101 channel allowed us to differentiate neurons from astrocytes and provided a constant intensity image for off-line motion correction (Dombeck et al., 2007). ICMS was then used (supplemental Fig. $1 B$, available at www. jneurosci.org as supplemental material) at each injection site in each mouse to verify the location as the forelimb motor cortex. The lightly anesthetized mouse ( $\sim 0.25-0.5 \%$ isoflurane) was held by the head-plate and tail such that its limbs were free to hang in space. Pipettes filled with $3 \mathrm{M} \mathrm{KCl}(\sim 1 \mathrm{M} \Omega)$ were advanced through the cortex such that the tip was in Layer $5(\sim 550 \mu \mathrm{m}$ deep $)$ directly below the dye injection sites. Cathodal pulse trains $(6-30 \mathrm{~ms}$ train duration, 100-400 $\mu \mathrm{A}, 400 \mu \mathrm{s}$ pulse duration, $333 \mathrm{~Hz}$ ) (Hewlett-Packard $8116 \mathrm{~A}$ pulse generator, A.M.P.I. Iso-flex) starting with low amplitude and short train durations were applied and slowly increased until contralateral forelimb muscle twitches could be observed. The ICMS used to determine the location of the motor area at each injection site took only a few minutes, involved one electrode penetration per site and typically involved $<\sim 7$ stimulus trains per site. All time-series were acquired from locations in which the movement was almost entirely from a region of the forelimb; occasionally a smaller twitch of the ipsilateral forelimb could be discerned, but no twitches of the face or other body regions were detectable.

The two-photon microscope used for imaging has been previously described (Dombeck et al., 2007), however the collection optics were modified: 680sp (Semrock) IR blocking filter, 542/50 (Semrock) calcium green channel emission filter, 610/75 (Chroma) SR101 channel emission filter. In most experiments, GaAsP PMTs (1077P-40, Hamamatsu) were used, but Multi-Alkali PMTs (R6357, Hamamatsu) were used in a few of the initial experiments. The illumination source was a Ti:Sapphire laser (Mira 900 or Chameleon Ultra II, Coherent, $\sim 880 \mathrm{~nm}$ excitation light, $\sim 100$ fs pulses at $80 \mathrm{MHz}$ ) and a $40 \times, 0.8 \mathrm{NA}$ objective (Olympus) was used in all experiments. Sixty-two time-series $(128 \times 128$ pixel $)$ from 20 different dye injection sites in 10 mice were analyzed. Four time-series were acquired at $4 \mathrm{~Hz}$ frame rate ( $2 \mathrm{~ms} /$ line, $500-1500$ frames) and 58 at $8 \mathrm{~Hz}$ ( $1 \mathrm{~ms} /$ line, $1000-2000$ frames); the field of view was $\sim 200 \mu \mathrm{m}$ with an average of $\sim 90$ neurons/image field. Because the decay time of action potential evoked somatic calcium transients under similar conditions to ours is typically many hundreds of milliseconds (Kerr et al., 2005), it is 
unlikely that any transients were missed with the $\sim 8 \mathrm{~Hz}$ frame rate used for almost all of our recordings (see supplemental Fig. 2, available at www.jneurosci.org as supplemental material). Though our frame rate is orders of magnitude slower than data acquisition rates using electrophysiological recording methods, it is common in the motor cortex to find average spike rates on a similar timescale as our effective recording rate for analysis and interpretation (Moritz et al., 2008). The resulting time course of firing rates look qualitatively similar to the activity traces obtained here from calcium-sensitive dye imaging (Moritz et al., 2008).

Complex motor pattern discrimination. Two optical computer mice were used to record spherical treadmill velocity (Dombeck et al., 2007). A running state vector was generated by first calculating three velocity components of ball motion by separately integrating the counts received at $5 \mathrm{~ms}$ intervals from the orthogonal motion sensors of each computer mouse. Speed was calculated as the Euclidean norm of 3 perpendicular components of ball velocity and the up state of the running state vector started when the speed was $>0.04 \mathrm{~m} / \mathrm{s}$ and returned to the down state when running speed was $<0.01 \mathrm{~m} / \mathrm{s}$. Running involved coordinated movements of all four limbs when all of the paws of the mouse were on or close to the surface of the treadmill and was defined to include the following components: running forward, running sideways or backing up. Running was typically spontaneous, but was occasionally elicited by using an airpuff to the mouse's flank as described previously (Dombeck et al., 2007). A grooming state vector was generated by hand while viewing the CCD camera movies frame-by-frame. Grooming was defined to include mouse forelimb (contralateral to the two-photon imaged motor cortex hemisphere) movements while the forelimb was off of the treadmill surface and the mouse was in the prone position; nearly all of these movements included one or more of the following components: forepaw licking, nose/face stroking, stroking of the whiskers, and/or forelimb fanning in front of the mouse. To increase the frequency of grooming, honey ( $\sim 1$ part honey:1 part water) was periodically applied to the whiskers.

Data analysis. Analysis was performed using ImageJ (1.40 g) and custom scripts written in MatLab (version 7). All data in the text and figures are presented as mean \pm SD, except in the plots shown in Figures 2 and 6 and supplemental Figure 3, available at www.jneurosci.org as supplemental material, where the error bars represent SE.

Our previously developed Hidden Markov Model line by line motion correction algorithm (Dombeck et al., 2007) was run on the static red SR101 channel of the two-photon time-series to determine the brain motion. This information was then used to shift the collected lines of each frame of the dynamic calcium-sensitive dye channel in the time series back to their correct position with respect to reference frames. The mean in plane $(X-Y)$ Euclidean distance brain motion during running, grooming and resting periods was $1.7 \pm 0.6 \mu \mathrm{m}, 2.3 \pm 0.9 \mu \mathrm{m}, 0.2 \pm 0.1$ $\mu \mathrm{m}$ respectively and the mean out of plane $(z)$ motion during running, grooming and resting periods was $0.7 \pm 0.2 \mu \mathrm{m}, 1.0 \pm 0.4 \mu \mathrm{m}, 0.5 \pm 0.2$ $\mu \mathrm{m}$ respectively. Neither the brain motion nor the motion correction algorithm induced any artificial spatial correlations that significantly contributed to the decrease in neuron-neuron correlation as a function of neuron-neuron distance result shown in Figure 2 (see supplemental Fig. 3, available at www.jneurosci.org as supplemental material). Polygonal regions of interest (ROI) were manually defined on the time projection image of the time-series to closely approximate the outline of the neuron of interest (neurons were defined as non-SR101 labeled cells). Fractional changes in fluorescence for a given frame were calculated on a pixel by pixel basis relative to the mean. The values for pixels present in the ROI after motion correction were averaged. Occasionally, brainmotion was such that no pixels were present in a specific ROI during a given frame; the neurons corresponding to these ROIs were excluded from all analysis. Slow time scale changes in the fluorescence time series were removed by examining the distribution of fluorescence in a $\pm 12.5 \mathrm{~s}$ interval around each sample time point and subtracting the $8 \%$ percentile value. For $\sim 90 \%$ of the neuron fluorescence traces, this method reliably subtracted slow changes without significantly filtering longer events that had distinct onsets and offsets; however, in $\sim 10 \%$ of the traces in which much longer duration transients were present, this method was found to alter the longer events and hence was not used.
Instead, the baseline for these neurons was defined by hand and then subtracted from the raw trace. The baseline subtracted neuron fluorescence traces were then subjected to the analysis of the ratio of positive to negative going transients of various amplitudes and durations that we described previously (Dombeck et al., 2007). We used this analysis to identify significant transients with $<5 \%$ false positive error rates and generated the significant transient only traces (see Figs. $1 B, 4 A$; orange traces) that were used for all analysis in this research. Note that the significant transient only traces are not discontinuous traces; the significant transients are left untouched, but the time points between the significant transients are all set to 0 . The point of generating the significant transient only traces was to eliminate the effects of any possible motion artifact induced negative going transients on our analysis. However, the effects of these artifacts was minimal (supplemental Fig. 3, available at www.jneurosci.org as supplemental material) and there was very little difference between our results generated with the raw or with the significant transient only traces (see supplemental Fig. $4 B, C$, available at www. jneurosci.org as supplemental material). Correlations were calculated as the Pearson's correlation coefficient between the two traces of interest. The statistical significance of the correlation values $(C)$ between fluorescence traces [either significant transient only single neuron fluorescence or mean cluster fluorescence (defined as the mean of the significant transient only traces of all of the neurons in a cluster)] and complex mouse movement state vectors was determined by bootstrapping as follows. Each fluorescence trace was broken into at least 9 segments (determined by the significant transients) that were randomly shuffled. A distribution of 10,000 random correlation values $\left(C_{\text {rand }}\right)$ was generated by calculating the correlation coefficient between each randomly shuffled fluorescence trace and the state vectors. The $p$ value for the significance of $C$ was calculated as the fraction of the total number of $C_{\text {rand }}$ with values greater than $C$. Neuron-neuron distances were calculated as the Euclidean distance between the ROI centroids of the two neurons.

The following was used to determine the direction of maximal physical separation between the running and grooming correlations of neurons. First, the running correlation weighted mean and SD of position of all of the neurons along a chosen direction was calculated; the positions of the neurons were weighted by their running correlation coefficients (only significant correlation values were considered). This calculation was then repeated to determine the grooming correlation weighted mean and SD of position of all of the neurons along the same direction. A $t$ test was then performed between the resulting running and grooming weighted position distributions to determine the $T$-statistic. This $T$-statistic is essentially a measure of whether any significant spatial separation exists along the chosen direction between running and grooming neuron correlation on the $\sim 200 \mu \mathrm{m}$ scale of the image fields. We calculated this T-statistic along 100 different directions (corresponding to angles from 0 to $180^{\circ}$ in $1.8^{\circ}$ increments) within each dataset image field. The direction (angle) corresponding to the maximum $T$-statistic was then selected. We refer to the maximum $T$-statistic as the spatial significance. The $T$-statistic for each dataset was converted into a $p$ value for the categorizations shown in Figure 5.

The meta-K-means algorithm was applied independently to each of the 62 image fields that were studied here. It employed 2500 runs of a traditional K-means algorithm that was seeded each run by using the K-means + + seeding strategy (Arthur and Vassilvitskii, 2007). Each run generated 4 clusters, and correlation was used as the measure that K-means minimized with respect to. Neurons that were in the same cluster in $>80 \%$ of the K-means runs were placed in metaclusters. Neurons that were not in the same cluster with any other neurons in $>80 \%$ of the K-means runs were disregarded; this occurred for $4.3 \pm 2.7$ neurons per dataset ( 265 out of 4813 total neurons). To form the final clusters, the metaclusters were then combined if the correlation between the mean fluorescence traces of all of the neurons in each metacluster was greater than a predetermined threshold $\left(T_{\text {corr }}\right)$. $T_{\text {corr }}$ could vary from image field to image field (i.e., each of the 62 fields could have a different value of $\left.T_{\text {corr }}\right)$. It was determined by running the above described meta-K-means algorithm many times, each time with a different value of $T_{\text {corr }}$. The wide range of $T_{\text {corr }}$ values $(0.45-0.95$, in steps of 0.025$)$ were typically sufficient to explore the full spectrum of possible metacluster combina- 
tions from generating many small clusters with $T_{\text {corr }}=0.95$ (leaving the metaclusters uncombined) to generating one or two large clusters with $T_{\text {corr }}=0.45$ (see supplemental Fig. 10 , available at www.jneurosci.org as supplemental material). A heuristic was developed to select the one specific value of $T_{\text {corr }}$ that simultaneously maximized both the mean cluster size and the weighted mean of the intracluster neuron-neuron correlations (weighted by cluster size). This value of $T_{\text {corr }}$ was the value that maximized the product of the mean cluster size (scaled from 0 to 1 ) as a function of $T_{\text {corr }}$ and the mean intracluster neuron-neuron correlation (scaled from 0 to 1 ) as a function of $T_{\text {corr }}$. This value of $T_{\text {corr }}$ was then used in the above described meta-K-means algorithm to generate the final clusters of neurons for each of the 62 image fields.

\section{Results}

Optical recording of calcium transients with cellular resolution in forelimb motor cortex of awake running and grooming mice

To study the functional clustering within the mouse motor cortex microcircuitry, we bolus loaded (Stosiek et al., 2003) Calcium Green-1-AM into layer 2/3 of the forelimb motor cortex (Fig. 1Ai). We acquired twophoton time-series movies of the labeled area while the mouse displayed the movements of running and grooming (Fig. 1Aii,iii) (see Materials and Methods for definitions of running and grooming). This provided a situation in which we could optically record the spatio-temporal activity of forelimb motor cortex neuron populations while the mouse's forelimb was used in two qualitatively different and temporally distinct rhythmic movements. During the acquisition of the two-photon time-series movies, the mice ran, groomed, and rested $38 \pm 15,21 \pm 14$, and $41 \pm 16 \%$ of the time on average, respectively. Calcium Green- $1 \Delta F / F$ traces (Fig. $1 B$, black traces with orange segments) were extracted from ROIs drawn around each neuron in the field. These $\Delta F / F$ traces revealed a baseline periodically interrupted by calcium transients that were typically characterized by a sharp onset followed by a slower decay. The calcium transients were of various amplitudes, consistent with a difference in the number of underlying action potentials (Kerr et al., 2005; Sato et al., 2007), and of various durations, consistent with the summation of multiple transients (Dombeck et al., 2007; Greenberg et al., 2008). Significant transients with $<5 \%$ false positive error rates were identified (see Materials and Methods) and used in all subsequent analysis (Fig. $1 B$, orange traces). These traces were taken as a surrogate measure of spiking activity and are referred to as the temporal activity pattern of the neurons. In all, 62 timeseries ( $\sim 200 \mu \mathrm{m}$ field of view, $87 \pm 14$ neurons/image field, $4-8 \mathrm{~Hz}$ frame rate) from 20 different dye injection sites in 10 mice were analyzed.

\section{Pairwise temporal correlation between neurons is inversely related to distance}

We began by studying in the awake behaving mouse whether neuron-neuron correlation varied as a function of the dis-

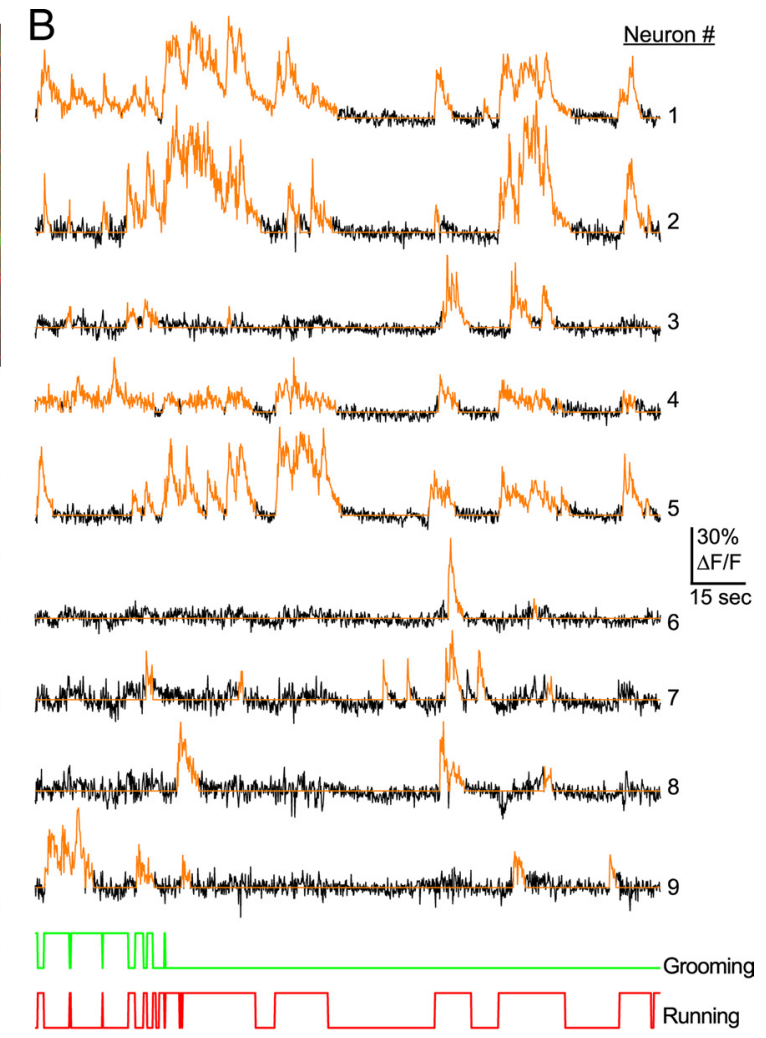

Figure 1. Optically recording calcium transients with cellular resolution in forelimb motor cortex of awake running and grooming mice. Ai, A two-photon microscopy (TPM) image of a typical field of view from a bolus loaded region of forelimb motor cortex spond calcium-sensitive dye. The red SR101 dy acquisition. The limbs of the mouse are resting on the spherical treadmill. One of the two horizontal head-restraint bars can be seen ttached to the head-plate and the microscope objective can be seen at the correct position used to image cortical layer $2 / 3$ through traces indicate significant calcium transients with $<5 \%$ false positive error rates (see Materials and Methods). The running and grooming state vectors are shown at the bottom.

tance between the neurons within our $\sim 200 \mu \mathrm{m}$ fields of view. For each time-series, we calculated all pairwise temporal activity pattern correlations and pairwise distances between neurons and then we combined the results from all 62 timeseries. When we plotted mean temporal correlation as a function of mean distance between the neurons (Fig. 2), we found a significant inverse relationship between the two (Spearman's rank correlation coefficient of $-0.9971, p<10^{-5}$ ) ( slope $=$ $-8.1 \times 10^{-4} / \mu \mathrm{m}, 95 \%$ confidence interval: $(-8.5 \times$ $\left.\left.10^{-4},-7.7 \times 10^{-4}\right) / \mu \mathrm{m}\right)$ : on average, the nearer neurons were to each other in physical space, the more similar were their activity patterns.

This statistical analysis of the correlation versus spatial separation between neurons within the forelimb motor cortex during movement (Fig. 2) demonstrates that the idea of "like attracts like" is an important notion for understanding the microorganization of the awake motor cortex, but it provides no specifics about the detailed topography of the area. How does the statistical spatial relationship between correlated neurons manifest itself in the real space of the brain? In the rest of the Results, we explore this question using two separate approaches to study the spatial order within our $\sim 200 \mu \mathrm{m}$ fields of view. We first describe a K-means algorithm that clusters neurons based on their temporal activity patterns and then we study the spatial 


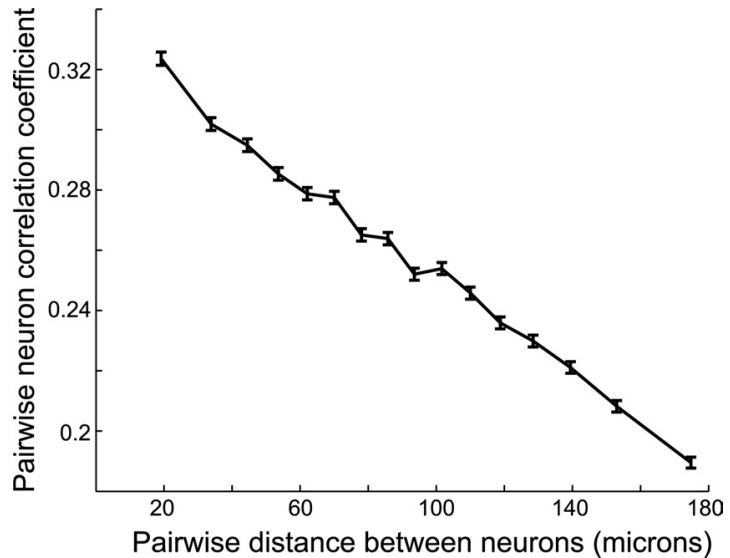

Figure 2. Plot of mean neuron-neuron temporal activity pattern correlation versus mean neuron-neuron distance averaged over all acquired time-series. The error bars represent SE.

arrangement of the clusters of neurons. Second, we code each neuron within an image according to the correlation of its temporal activity pattern to running and/or to grooming and then examine the spatial layout of the coded neurons. While any spatial clustering determined by either approach is susceptible to some degree of parameter dependence (K-means analysis for the selection of specific thresholds; mouse movement correlation analysis for the selection of the important aspects of forelimb movement, see below), in the end we find that the two approaches spatially segment the neurons very similarly, adding confidence to the end results.

\section{Temporally correlated neurons cluster into spatially distinct groups}

We used a clustering technique to examine if neurons could be clustered solely by the correlations between their temporal activity patterns, and if so, if the clusters exhibited any spatial organization. Here we describe our clustering algorithm by first showing the clustering (Fig. $3 A$ ) and spatial organization (Fig. $3 C)$ results on one specific example dataset and then by showing the combined clustering (Fig. 3B) and spatial organization (Fig. $3 D, E)$ results from all 62 datasets. We adapted a meta-K-means algorithm (Ozden et al., 2008) to separate the activity traces of the neurons within each time-series into discrete clusters. The algorithm started with the individual neuron activity traces (Fig. 3Ai) which were arranged randomly with respect to their neuronneuron correlation values (Fig. 3Aii). The algorithm employed numerous runs of $\mathrm{K}$-means ++ , which is a carefully seeded version of the traditional K-means algorithm (Arthur and Vassilvitskii, 2007), followed by a series of steps designed to maximize both the cluster size and intracluster neuron-neuron correlations (see Materials and Methods for details). It is unlikely that our algorithm (or any other K-means algorithm) finds the optimal clustering of the neural activity traces (a computationally NP-hard problem), but it was successful in segmenting the data in a reasonable manner. When the algorithm was run on the activity traces shown in the example of Figure $3 A i$, it found two large clusters (Fig. 3Aiii,iv, clusters 1 and 2) and four smaller clusters (Fig. 3Aiii, iv, other clusters); for display in Figure 3, Aiii and Aiv, the traces were sorted first into their respective clusters and then within each cluster by their correlation to the cluster's mean activity trace (see supplemental Fig. 5, available at www.jneurosci. org as supplemental material, for the same plot shown in Fig. 3Aiii, but with the traces not sorted within each cluster). This sorting makes it apparent for this example that the intracluster neuron activity patterns were more similar than intercluster neuron activity patterns (Fig. 3Aiii), and that the intracluster neuron-neuron correlations were significantly larger than intercluster neuronneuron correlations $\left(0.40 \pm 0.14\right.$ vs $0.18 \pm 0.16, p<10^{-6}$, twotailed $t$ test, $N=86$ neurons) (Fig. 3Aiv). In general, the combined results of the meta-K-means clustering on all of the time-series datasets revealed that the algorithm consistently found clusters with mean intracluster neuron-neuron correlations greater than the intercluster neuron-neuron correlations $\left(0.43 \pm 0.18\right.$ vs $0.15 \pm 0.17, p<10^{-10}$, two-tailed $t$ test, $N=4813$ neurons). When clusters of the same size were generated by randomly assigning neurons to a cluster, the mean intracluster neuron-neuron correlation $(0.31 \pm 0.23)$ was between, and statistically different from, the intercluster $\left(p<10^{-10}\right.$, twotailed $t$ test, $N=4813$ neurons $)$ and intracluster $\left(p<10^{-10}\right.$, two-tailed $t$ test, $N=4813$ neurons) neuron-neuron correlation values obtained by the algorithm (Fig. $3 B$ ).

With all of the neurons within a field of view assigned to a cluster based on the correlations between their activity patterns, we could then examine the spatial micro-organization of the clustered neurons by looking at their physical locations with respect to each other. Figure $3 \mathrm{Ci}$ shows the field of view from which the neural activity traces in Figure 3Ai,iii were acquired. When the neurons within this field were false-colored according to their cluster identity, a clear spatial separation was observed (Fig. 3Cii); most striking is the separation between the largest clusters, clusters 1 and 2 (Fig. 3Cii, red- and green-colored clusters, respectively). The spatial separation between the clustered neurons within the field of this specific example was statistically significant, with the mean intracluster neuron-neuron distance less than the mean intercluster neuron-neuron distance ( $86 \pm 42$ vs $107 \pm 48 \mu \mathrm{m}, p<0.01$, two-tailed $t$ test, $N=86$ neurons). Due to the relatively large spatial extent of each of the large clusters (clusters 1 and 2), this small but significant difference between the intra- and intercluster neuron-neuron distances is exactly what is expected, even for the well spatially separated clusters shown in Figure 3Cii. Upon inspection, we found that many of the 62 fields of view contained spatially separated clusters such as those seen in Figure 3Cii. This effect of intercluster neuron-neuron distances being greater than intracluster distances was consistent when examined in each of the 62 datasets (Fig. 3E). When the results from all of the time-series datasets were combined, an average effect similar to that seen in the specific example of Figure 3, A and $C$, was observed: the mean intracluster neuron-neuron distances were smaller and significantly different from the mean intercluster neuron-neuron distances ( $88 \pm 43$ vs $95 \pm$ $44 \mu \mathrm{m}, p<10^{-6}$, two-tailed $t$ test, $N=4813$ neurons) (Fig. $3 D$ ). These results indicate that functional spatial clustering exists in the forelimb motor cortex of the awake mouse during movements such that coactive neurons are often clumped together. These highly internally correlated and often spatially distinct clusters are, at least partly, responsible for the inverse relationship seen between neuron-neuron correlation and distance in Figure 2.

\section{Neuronal clusters and individual neurons showed strong correlation to running and grooming}

We next studied whether the mean activity of the neuronal clusters had any correlation to the most apparent ongoing movements: running and grooming. Figure $4 A$ (bottom) shows an example of the mean temporal activity pattern for the largest clusters (clusters 1 and 2) from the specific example shown in 
Figure 3, $A$ and $C$, along with the timing of running and grooming movements. When the correlation of mean cluster activity to running or grooming was calculated (see supplemental Fig. 6A for data displayed in a two dimensional plot, available at www.jneurosci.org as supplemental material), it was apparent that the largest clusters were significantly correlated to running (cluster 1 , running correlation $=$ 0.60 , grooming correlation $=-0.10$ ) and grooming (cluster 2, grooming correlation $=0.61$, running correlation $=$ $-0.07)$, while the other smaller clusters had little correlation to these two movements. Upon inspection, it became apparent that many of the clusters in our datasets had mean temporal activity patterns that closely matched the time course of running, grooming, or both movements, but far fewer clusters had activity patterns that matched only specific components of the movements (such as stroking the whiskers or running only forward, see Materials and Methods for more details on the components) or different more subtle movements not relating to running or grooming. Therefore, when examined across all of the datasets, a vast majority of the mean activity patterns of the large clusters were significantly correlated to running or grooming movements (supplemental Fig. 6B, available at www. jneurosci.org as supplemental material). In fact, $87.7 \%$ of the 4813 neurons in all 62 time-series were in clusters with statistically significant running and/or grooming correlation ( $p<0.05$; $p$ value from bootstrapping, see Materials and Methods). No large clusters (only small clusters containing $12.3 \%$ of the total number of neurons) were found that were not significantly correlated to the complex motor patterns of running and grooming $(p>0.05)$.

The results above demonstrate that the meta-K-means analysis is, in fact, segmenting the neurons into largely running and/or grooming correlated groups; it was therefore of interest to investigate running and grooming correlation on the single-cell level. A large fraction of individual neuron temporal activity patterns were correlated to running and grooming movements. This can be seen in the single neuron activity pattern traces of the example dataset shown in Figure $4 A$ (top) (Fig. 4 Bii, labeled neurons; time-series dataset same as Fig. $3 A, C$ ) and in the combined data of the 4813 neurons from all 62 datasets (supplemental Fig. 6C, available at www.jneurosci.org as supplemental material), where $82.9 \%$ of individual neurons had statistically significant running and/or grooming correlations ( $p<0.05$, $p$ value from bootstrapping). Because of this large fraction of individual neurons with statistically significant running and/or grooming correlation, we
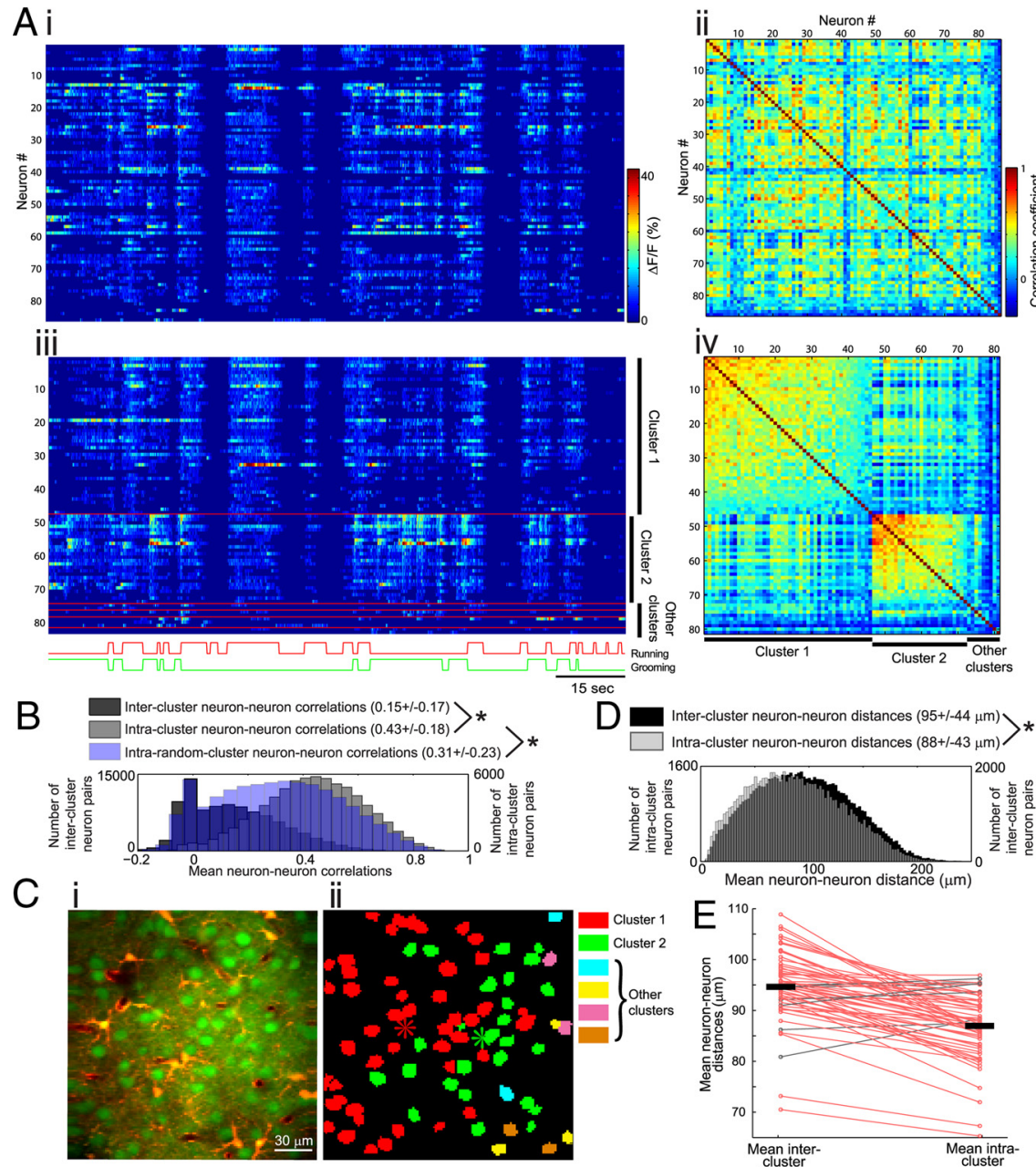

Figure 3. Meta-K-means clustering of neurons based on their temporal activity patterns. $A$, A plot of neuron temporal activity patterns (Ai) and neuron-neuron correlations (Aii) for the 86 neurons in the field of view shown in $\mathbf{C}$. The ordering of the neurons in $\mathbf{A i}$ and $\boldsymbol{A i i}$ is random. After meta-K-means clustering, the neurons were grouped into clusters based on their temporal activity patterns (Aiii). The neurons were sorted first into their respective clusters and then within each cluster by their correlation to the cluster's mean activity trace. This sorting is most apparent in the neuron-neuron correlation plot (Aiv). Note that 5 of the neurons were not assigned to a cluster due to their dissimilar activity patterns compared to all other neurons and therefore they are not shown in Aiiii and Aiv. Though the running and grooming state vectors were not used in the meta-K-means clustering, they are shown at the bottom of $\boldsymbol{A i i i i ~ f o r ~ r e f e r e n c e ~ i n ~} \boldsymbol{A}$ i and $\boldsymbol{A i i i}$. $\boldsymbol{B}$, Histogram of intra- and intercluster neuron-neuron correlation values for all 62 time-series datasets (light gray and dark gray, respectively) along with intracluster neuron-neuron correlation values for the same cluster sizes but with randomly assigned neurons (purple). ${ }^{*} p<$ $10^{-10}$. Ci, TPM image of the field of view containing the 86 neurons from $\boldsymbol{A}$. Cii, False color image of the field shown in $\boldsymbol{C i}$ in which the neurons are colored according to their cluster identity. A red or green asterisk has been placed at the mean position of all of the neurons in cluster 1 or cluster 2 , respectively. The mean $x$-position of the neurons in cluster 1 is significantly different $\left(p<10^{-5}\right.$, two-tailed $t$ test, $N=46$ neurons in cluster 1 and 26 neurons in cluster 2) from the mean $x$-position of the neurons in cluster 2 , while the $y$-positions are not significantly different $(p=0.56)$. D , Histogram of the intra- and intercluster neuron-neuron distances for all 62 time-series datasets (light gray and black, respectively). ${ }^{*} p<10^{-6} . \boldsymbol{E}$, A line plot with one line for each dataset going from the mean intercluster distance to the mean intracluster distance. Lines with negative and positive slope are colored red and black respectively. The black bars represent the mean neuron-neuron distance. Note that the majority of the lines are red, indicating that for the majority of the 62 datasets, the intercluster neuron-neuron distances are greater than the intracluster neuron-neuron distances.

next investigated the spatial clustering of running and grooming correlation on the single-cell level. When the neurons within the image from the example time-series dataset shown in Figure 3, A and $C$, were coded according to the magnitude of their running and/or grooming correlation, it became clear that the resulting spatial segmentation of the field was nearly identical to that obtained using the meta-K-means analysis (Fig. $4 \mathrm{Bi}, \mathrm{ii}$ ). Therefore, the spatial segmentation of the neurons provided by meta-Kmeans (knowing nothing about the running or grooming time course) are very similar, and in some cases nearly identical, to the 

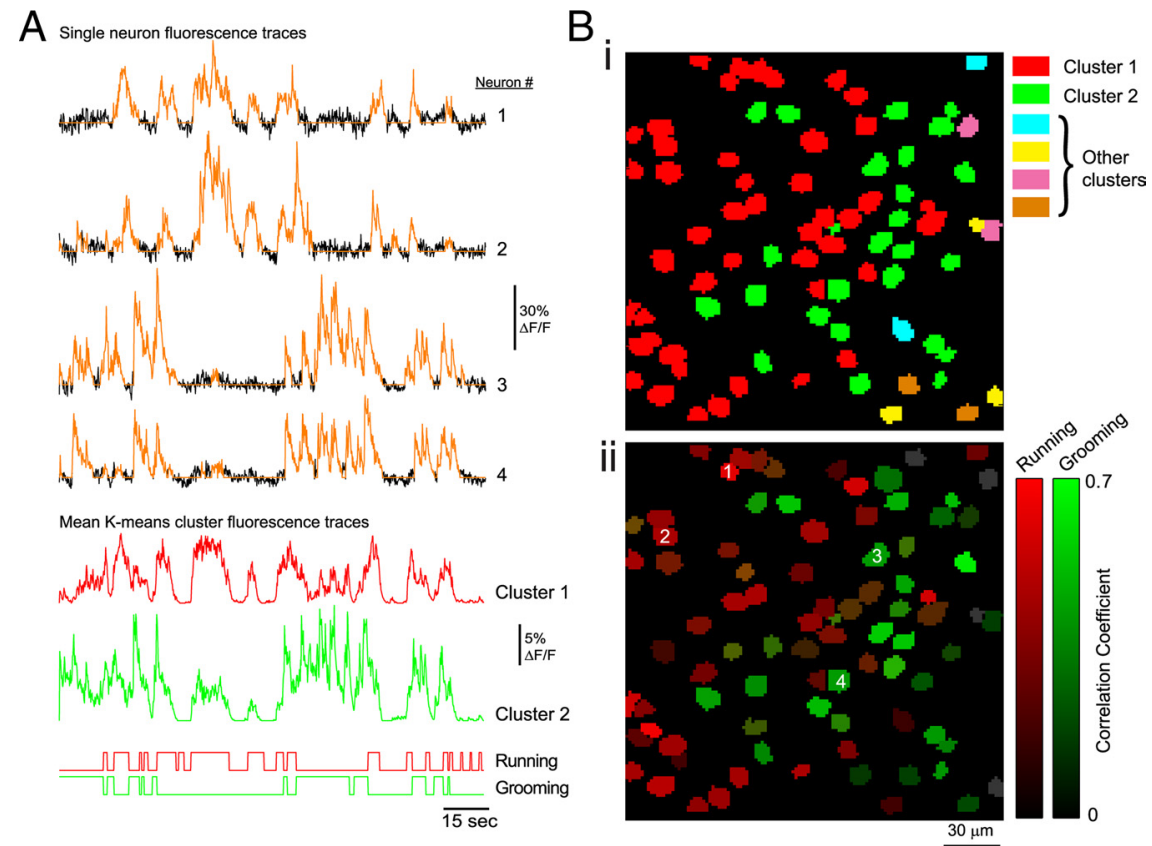

Figure 4. Neuronal clusters and individual neurons showed strong correlation to running and grooming. $\boldsymbol{A}$, Bottom, Mean temporal activity pattern of all of the neurons in cluster 1 (red) and cluster 2 (green) from Figure 3 Aiii, iv, (ii along with the running and grooming state vectors. Top, Single neuron temporal activity pattern traces (orange) (Calcium green-1 fluorescence versus time traces shown in black) for the neurons labeled in Bii; running and grooming state vectors same as bottom. Neurons 1 and 2 are running correlated (running correlations $=0.63$ and 0.47 , grooming correlation $=-0.37$ and -0.26 , respectively); Neurons 3 and 4 are grooming correlated (grooming correlation $=0.43$ and 0.43 , running correlations $=-0.13$ and -0.12 , respectively). $B$, Comparison of the two different methods of segmenting the neurons within a field (dataset same as Fig. $3 A, C$ ): $i$, neurons are colored according to their cluster identity obtained from meta-K-means analysis of their temporal activity patterns, and $i i$, neurons are colored according to the magnitude of their correlation to running (red) and grooming (green); only significant $(p<0.05$ ) positive correlation values are shown.

spatial segmentation based on correlations between single neuron activity and mouse movements.

We note that because many different low-level aspects of forelimb movement could be used to distinguish running from grooming (muscle activation, hand position with respect to the body, speed or direction of hand movements, etc.), it is unclear which aspect is most important for segmenting the neurons. Although we refer to neurons here as running correlated or grooming correlated, this terminology is not meant to imply a strict ethological function for the neurons and is meant to encompass any of the possibly important aspects of forelimb movement.

\section{The spatial layout of neurons with temporal activity patterns correlated to running or grooming}

To study the spatial layout of the movement correlated neurons, we coded the neurons within an image according to the magnitude of their running and grooming correlations and then determined the direction along which the maximum physical separation between running and grooming correlations occurred (see Materials and Methods) (Fig. 5, white arrows). We refer to this measure of physical separation as the spatial significance. The distribution of spatial significance values from all time-series datasets (excluding the 5 homogeneous fields, defined below) is shown in supplemental Figure $7 A$, available at www.jneurosci.org as supplemental material, along with the spatial significance values obtained from the datasets with the neurons in the same physical location, but with their correlation coefficients randomly drawn from the distribution in supplemental Figure 6C, available at www. jneurosci.org as supplemental material; the data showed a signif- icantly greater level of spatial significance compared to the randomized data $(2.1 \pm$ 1.3 vs $1.3 \pm 0.7, p<10^{-13}$, two-tailed $t$ test, $N=57$ image fields, $M=310$ fields with randomized correlations). We categorized each of the 62 time series datasets into one of four possible categories and show 2 examples of each in Figure 5: homogeneous (Fig. 5A), significant spatial separation (spatial significance $p<0.01$ ) (Fig. 5B) (see supplemental Movie 2, available at www.jneurosci.org as supplemental material), moderate spatial separation (spatial significance $0.01<p<$ 0.1 ) (Fig. 5C), and little spatial separation (spatial significance $p>0.1$ ) (Fig. 5D).

Homogeneous image fields are characterized by the presence of only one movement correlated neuron type throughout the field. These fields were relatively rare, consisting of only $\sim 8 \%$ of the total number of fields investigated. Significant spatial separation image fields are characterized by the presence and clear spatial separation of both correlation types. Often, these fields appeared as border regions between two homogeneous fields, or a large homogeneous field containing a smaller embedded homogeneous field of the opposite movement correlated neuron type. These fields consisted of $\sim 34 \%$ of the total number of image fields. Moderate spatial separation fields $(\sim 32 \%$ of the total number of fields) consisted of both neuron correlation types and, while some spatial separation between the correlation types was observed, the two populations were more intermingled compared to the significant spatial separation image fields. The image fields with little spatial separation $(\sim 26 \%$ of the total number of fields) between the neuron correlation types often appeared to have the two populations randomly mixed: no spatial clustering could be discerned on the $\sim 200 \mu \mathrm{m}$ scale. It is possible that many of these little separation fields consist of smaller significant subdomains of running or grooming correlated neurons for which the larger scale spatial order disappears. However, this was not seen; not only was no spatial significance observed on the $\sim 200 \mu \mathrm{m}$ scale, it was also not observed on any smaller scale (supplemental Fig. 7Bii, available at www. jneurosci.org as supplemental material). Interestingly, when we examined the mean spatial significance of all of the datasets (excluding the homogeneous fields) on a scale smaller than $\sim 200 \mu \mathrm{m}$, we found spatial significance down to a scale of $\sim 100 \mu \mathrm{m}(p<0.01$, two-tailed $t$ test, $N=57$ datasets, $M=570$ randomized datasets) (supplemental Fig. 7Bi, available at www.jneurosci.org as supplemental material), indicating that functional clustering within this region of the cortex is present above this spatial scale, but not below it.

To investigate the contribution that spatially segmented running and grooming neuron correlations may have on the inverse relationship seen between neuron-neuron correlation and distance in Figure 2, we plotted neuron-neuron correlation versus distance first for all of the significant spatial separation fields and then for the all of the little spatial separation fields (Fig. 6A). While both subsets showed a significant inverse relationship be- 

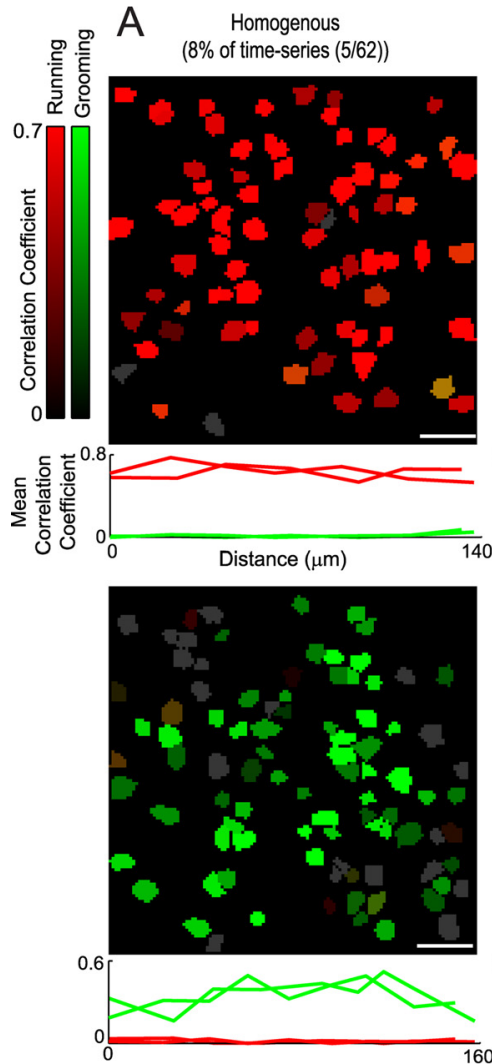

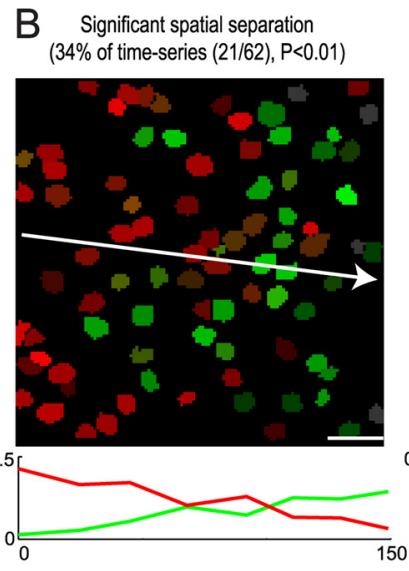

C Moderate spatial separation (32\% of time-series $(20 / 62), 0.01<\mathrm{P}<0.1)$
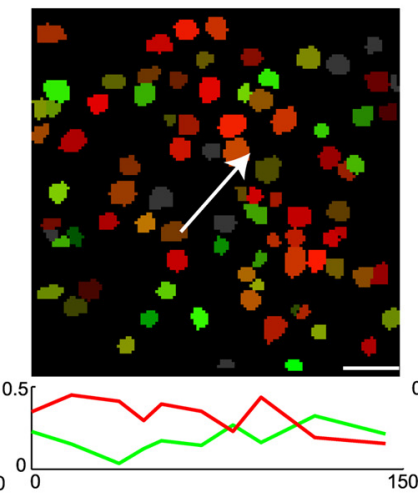

Little spatial separation (26\% of time-series (16/62), $0.1<P$ )

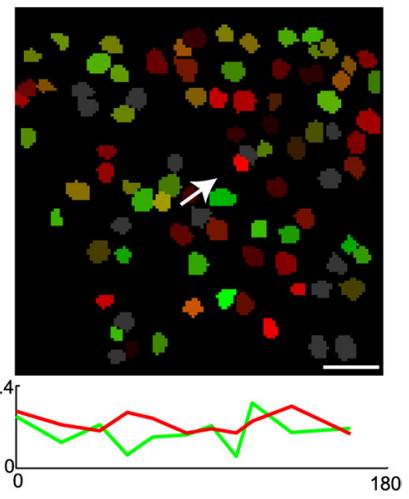

Figure 5. The spatial layout of neurons with temporal activity patterns correlated to running or grooming. When the neurons within each image were colored according to the magnitude of their movement correlations, it was possible to categorize the time-series fields based on their spatial significance value: Homogeneous ( $\boldsymbol{A}$, top and bottom), significant spatial separation ( $\boldsymbol{B}$, top and bottom), moderate spatial separation ( $\boldsymbol{C}$, top and bottom), or little spatial separation ( $\boldsymbol{D}$, top and bottom). The arrows point in the direction of maximum physical separation between running and grooming correlations and the length of the arrows are directly related to the magnitude of the physical separation (the spatial significance). The plots associated with each image shows the mean neuron correlation to running (red) and grooming (green) as a function of distance along this maximum separation direction. Note that the maximum separation angle and spatial significance is poorly defined for a homogeneous field and therefore no arrows are shown in $\boldsymbol{A}$. Two perpendicular directions were chosen to generate the plots associated with each image in $\boldsymbol{A}$. Also note that only significant $(p<0.05)$ positive correlation values are shown. Scale bars: $30 \mu \mathrm{m}$.
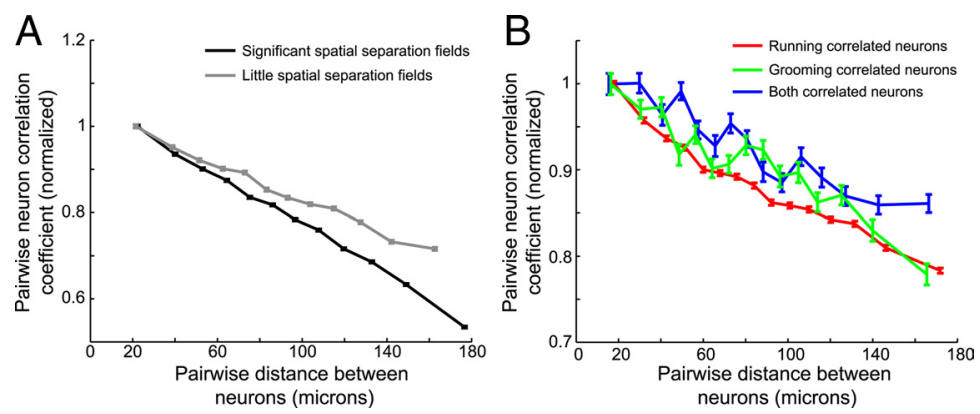

Figure 6. Plot of mean neuron-neuron temporal activity pattern correlation versus mean neuron-neuron distance averaged for subsets of the data: $\boldsymbol{A}$, all significant spatial separation fields (black) and all little spatial separation fields (gray); $\boldsymbol{B}$, all grooming (green, defined as $C_{\text {groom }}>0.3$ and $C_{\text {run }}<0.5^{*} C_{\text {groom }}$ ), all running (red, defined as $C_{\text {run }}>0.3$ and $C_{\text {groom }}<0.5^{*} C_{\text {run }}$ ) or all both (blue, defined as $C_{\text {run (or groom) }}>0.3$ and $C_{\text {run (or groom) }}>C_{\text {groom (or run) }}>0.5^{*} C_{\text {run (or groom) }}$ ) movement correlated neurons from all 62 time-series datasets. The error bars represent SE. The traces have been normalized by the correlation value at the shortest pairwise distance. Note that for $A$, the significant spatial separation trace was normalized by 0.28 (the correlation value at $\sim 20$ $\mu \mathrm{m}$ ) and the little spatial separation trace was normalized by 0.33 , meaning that the little spatial separation fields have an overall higher level of neuron-neuron correlation compared to the significant spatial separation fields.

tween correlation and distance (Spearman's rank correlation coefficient of $-1, p<10^{-20}$ for both subsets), the slope for the significant separation fields (slope $=-8.1 \times 10^{-4} / \mu \mathrm{m}, 95 \%$ confidence interval: $\left.\left(-8.5 \times 10^{-4},-7.7 \times 10^{-4}\right) / \mu \mathrm{m}\right)$ was significantly less than $\left(p<10^{-4}\right.$, two-tailed $t$ test, $N=20$ degrees of freedom) the slope for the little separation fields (slope $=-6.5 \times$
$10^{-4} / \mu \mathrm{m}, \quad 95 \%$ confidence interval: $\left.\left(-7.0 \times 10^{-4},-6.0 \times 10^{-4}\right) / \mu \mathrm{m}\right)$. This indicates that the spatial separation seen between running and grooming correlations in the significant separation fields (and seen between many of the spatially distinct K-means clusters above) is part of the origin of the correlation versus distance result from Figure 2. Interestingly, the little separation fields showed a significant inverse relationship between neuron-neuron correlation and distance (Fig. $6 \mathrm{~A}$ ), indicating that spatial clustering was likely also present within the running or grooming correlation subpopulations. This was in fact the case, when we plotted correlation versus distance, a significant inverse relationship was found for each of the subpopulations (Spearman's rank correlation coefficient of $-0.985(p<$ $\left.10^{-5}\right),-0.921\left(p<10^{-6}\right),-0.954(p<$ $10^{-5}$ ) for the running, grooming, and both subpopulations, respectively) (Fig. $6 \mathrm{~B}$ ). This indicates that, in addition to spatial separation between running and grooming neuron correlations, spatial clustering within the running and grooming correlation subpopulations was also part of the origin of the correlation versus distance result from Figure 2. 


\section{Discussion}

We explored the correlation versus distance relationship between neurons within the forelimb motor cortex of awake mice during the movements of running and grooming by optically recording neuronal activity at cellular resolution. By imaging fields of $\sim 200$ $\mu \mathrm{m}$ in diameter, we were able to investigate $\sim 90$ layer $2 / 3$ neurons simultaneously and show statistically that the temporal correlation between neurons increased the closer the neurons were to each other (Fig. 2). We further explored this result by using two separate methods to spatially segment the neurons within each imaging field. First, we used a K-means analysis method that separated the neurons within an image field into clusters with similar activity patterns. When we looked at the spatial organization of the neurons within these clusters, we found that neurons with like activity patterns were often clumped together and were more likely to be nearer to each other than they were to neurons with dissimilar activity patterns. Second, we examined the spatial layout of single neurons that were coded according to their correlation to running or grooming. In the end, the two methods segmented the neurons very similarly: the meta-K-means analysis was segmenting the neurons into largely running and/or grooming correlated clusters. When we searched for spatial separation between running and grooming neuron correlations, we found that a majority of the fields had a moderate to significant level of spatial separation; this separation accounted for some, but not all, of the correlation versus distance result seen in Figure 2. Another component of the correlation versus distance result was an organization that existed within the clusters: the closer the neurons were to each other the more correlated they were. Therefore, the origin of the inverse relationship between correlation and distance seen statistically in the awake motor cortex (Fig. 2) was twofold: clusters of highly correlated neurons were often spatially distinct from one another and (even when the clusters were spatially intermingled) within the clusters, the more correlated the neurons were to each other, the shorter the distance between them. In general, our results suggest the existence of a functional spatial clustering of the population code within the awake mouse motor cortex microcircuitry (for a more global view see supplemental Fig. 9, available at www.jneurosci.org as supplemental material).

Few in vivo rodent studies have investigated the functional organization of the motor cortex, and none at the microscopic scale investigated here. In the existing studies, short duration ICMS has revealed blurred somatotopy representations on the $\sim 0.5-1 \mathrm{~mm}$ scale (Li and Waters, 1991; Pronichev and Lenkov, 1998), while longer duration ICMS in rats have shown spatially distinct $\sim 0.5-1 \mathrm{~mm}$ scale complex movement areas (Ramanathan et al., 2006). In a separate study in which brain slices were made from the forelimb motor cortex of mice, the local excitatory connectivity was probed on the $>\sim 100 \mu \mathrm{m}$ scale using laser-scanning photostimulation (Weiler et al., 2008). This study found the layer $2 / 3$ to layer 5 connections to be the dominant excitatory pathway, though it was also shown that multiple other intralaminar pathways were present, with layer 2 as the strongest intralaminar pathway. While direct somatodendritic stimulation precluded study of the local connectivity at $<\sim 100 \mu \mathrm{m}$, it was apparent that within layer $2 / 3$ the majority of intralaminar excitatory input comes from the local circuitry $(<500 \mu \mathrm{m})$ and the synaptic connection between neurons increases with decreasing distance (Weiler et al., 2008). In our current research, it is unclear whether the functional microclustering in awake animals is due to distant converging afferents or more local connectivity. The results of Weiler et al. show clear local connectivity on the scale of and in the same cortical region of our imaging experiments, thus demonstrating that it is likely an important component for understanding the microclustering that we observed within the awake mouse motor cortex.

Compared to the rodent, a far greater number of studies have investigated functional clustering within the primate motor cortex. However, functional imaging studies at the microscopic scale in awake subjects have only been performed in a handful of studies and thus far only in rodents (Dombeck et al., 2007; Flusberg et al., 2008; Greenberg et al., 2008). Therefore it is not yet possible to compare our functional microclustering results in the mouse to any primate functional microscopic imaging studies. Electrode studies in the primate motor cortex, however, have indirectly revealed direction columns on the scale of $\sim 100 \mu \mathrm{m}$ (Georgopoulos et al., 2007), while other studies have found muscle columns on the scale of 0.5-1 mm (Asanuma, 1975). Larger zones of functional organization $(2-5 \mathrm{~mm})$ were found when studying the ethological layout of the motor cortex (Graziano et al., 2002, 2005). It is unclear whether any correspondence can be made between the microcircuitry within the cortex of rodents and that within higher mammals. In a single neuron resolution imaging study of the visual cortex of anesthetized subjects for example, heterogeneous fields of orientation responsive neurons were found in rodents whereas highly structured homogeneous fields were observed in cats (Ohki et al., 2005). However, if we assume that our results from the mouse can be compared directly to research performed in the primate motor cortex, then two hypotheses can be formed concerning the possible functional microclustering within the primate motor cortex. The first assumes that the only differences between the motor cortices of primates and mice is their physical size (primate motor cortex is $\sim 6$ times larger than mouse motor cortex), and that any microstructure therein scales accordingly. This implies that functional clustering would exist in the primate motor cortex on the scale larger than $\sim 1 \mathrm{~mm}$, similar to the scale inferred from some electrode penetration studies (Asanuma, 1975; Graziano et al., 2002, 2005). On the other hand, it could be assumed that the physical size of cortical microcircuits is a fundamental building block of cortical hierarchy that does not scale with brain size. This would imply that functional clustering would exist in the primate motor cortex on the $>\sim 100 \mu \mathrm{m}$ scale that we observed in the mouse, similar to the scale inferred in a recent electrode study (Georgopoulos et al., 2007).

It is unclear what specific aspects of the running and grooming movements in our research are spatially clustered. Previous studies found functional clustering in the motor cortex in the form of subregions that emphasize control of complex movements (Graziano et al., 2002), direction of movements (Georgopoulos et al., 1982), and muscle activation (Asanuma, 1975). In a correlative study in which neuronal activity and behavior are simultaneously measured and compared, it is notoriously difficult to draw causal conclusions about the particular aspects of movement directly controlled by motor cortex neurons. Here, due to the correlative nature of our experiments, no definite conclusions can be made about which aspect of the mouse's complex behavior was most important for the functional spatial clustering.

Though we have focused on the forelimb motor cortex, it would also be of interest to investigate the spatial clustering in neighboring regions during running and grooming. For example, the use of the hind-limb is qualitatively different during the locomotion movements of running compared to the balancing 
movements needed during grooming. We hypothesize that due to the different muscle activations of the hind-limb these two movements, similar results to what we found in the forelimb motor cortex may be found. Wide-field imaging methods (Berger et al., 2007; Ferezou et al., 2007) may be useful to observe the large-scale activation of the motor cortex during running and grooming. These studies may help inform the locations to further probe in future two-photon imaging studies. Finally, we note that new techniques that have been applied to awake behaving rodents (Brecht et al., 2004; Ferezou et al., 2007; Kuhn et al., 2008; Poulet and Petersen, 2008) are likely to compliment our results. For example, it was recently found during periods of quiet wakefulness that layer $2 / 3$ barrel cortex neurons undergo $\sim 10 \mathrm{mV}$ amplitude Up-Down state transitions at $\sim 2-5 \mathrm{~Hz}$ (Poulet and Petersen, 2008). While the temporal resolution of our recordings was fast enough to see the $\sim 2-5 \mathrm{~Hz}$ transitions, subthreshold activity may not be detectable with our imaging method. We analyzed the significant calcium transients during periods of resting and found the rate of their occurrence $(0.29 \pm 0.14$ significant transients/s) to be an order of magnitude lower than the transitions recently observed (Poulet and Petersen, 2008). However, since more work is necessary to quantify the sensitivity of our method to this subthreshold activity, our inability to see these transitions during resting does not imply that they are not present; future experiments, possibly using different methods (Berger et al., 2007; Kuhn et al., 2008), will be needed to search for these signals in the motor cortex.

\section{References}

Aflalo TN, Graziano MS (2006) Possible origins of the complex topographic organization of motor cortex: reduction of a multidimensional space onto a two-dimensional array. J Neurosci 26:6288-6297.

Amirikian B, Georgopoulos AP (2003) Modular organization of directionally tuned cells in the motor cortex: is there a short-range order? Proc Natl Acad Sci U S A 100:12474-12479.

Arthur D, Vassilvitskii S (2007) K-means++: the advantages of careful seeding. Paper presented at the Symposium on Discrete Algorithms, New Orleans, LA, January.

Asanuma H (1975) Recent developments in the study of the columnar arrangement of neurons within the motor cortex. Physiol Rev 55:143-156.

Asanuma H, Rosén I (1972) Topographical organization of cortical efferent zones projecting to distal forelimb muscles in the monkey. Exp Brain Res 14:243-256.

Berger T, Borgdorff A, Crochet S, Neubauer FB, Lefort S, Fauvet B, Ferezou I, Carleton A, Lüscher HR, Petersen CC (2007) Combined voltage and calcium epifluorescence imaging in vitro and in vivo reveals subthreshold and suprathreshold dynamics of mouse barrel cortex. J Neurophysiol 97:3751-3762.

Brecht M, Schneider M, Sakmann B, Margrie TW (2004) Whisker movements evoked by stimulation of single pyramidal cells in rat motor cortex. Nature 427:704-710.

Cherniak C (1994) Component placement optimization in the brain. J Neurosci 14:2418-2427.

Chklovskii DB, Schikorski T, Stevens CF (2002) Wiring optimization in cortical circuits. Neuron 34:341-347.

Cowey A (1979) Cortical maps and visual perception: the Grindley Memorial Lecture. Q J Exp Psychol 31:1-17.

Dombeck DA, Khabbaz AN, Collman F, Adelman TL, Tank DW (2007) Imaging large-scale neural activity with cellular resolution in awake, mobile mice. Neuron 56:43-57.

Ferezou I, Haiss F, Gentet LJ, Aronoff R, Weber B, Petersen CC (2007) Spa- tiotemporal dynamics of cortical sensorimotor integration in behaving mice. Neuron 56:907-923.

Flusberg BA, Nimmerjahn A, Cocker ED, Mukamel EA, Barretto RP, Ko TH, Burns LD, Jung JC, Schnitzer MJ (2008) High-speed, miniaturized fluorescence microscopy in freely moving mice. Nat Methods 5:935-938.

Georgopoulos AP, Kalaska JF, Caminiti R, Massey JT (1982) On the relations between the direction of two-dimensional arm movements and cell discharge in primate motor cortex. J Neurosci 2:1527-1537.

Georgopoulos AP, Merchant H, Naselaris T, Amirikian B (2007) Mapping of the preferred direction in the motor cortex. Proc Natl Acad Sci U S A 104:11068-11072.

Graziano MS, Aflalo TN (2007) Mapping behavioral repertoire onto the cortex. Neuron 56:239-251.

Graziano MS, Taylor CS, Moore T (2002) Complex movements evoked by microstimulation of precentral cortex. Neuron 34:841-851.

Graziano MS, Aflalo TN, Cooke DF (2005) Arm movements evoked by electrical stimulation in the motor cortex of monkeys. J Neurophysiol 94:4209-4223.

Greenberg DS, Houweling AR, Kerr JN (2008) Population imaging of ongoing neuronal activity in the visual cortex of awake rats. Nat Neurosci 11:749-751.

Kerr JN, Greenberg D, Helmchen F (2005) Imaging input and output of neocortical networks in vivo. Proc Natl Acad Sci U S A 102:14063-14068.

Kerr JN, de Kock CP, Greenberg DS, Bruno RM, Sakmann B, Helmchen F (2007) Spatial organization of neuronal population responses in layer 2/3 of rat barrel cortex. J Neurosci 27:13316-13328.

Klyachko VA, Stevens CF (2003) Connectivity optimization and the positioning of cortical areas. Proc Natl Acad Sci U S A 100:7937-7941.

Kuhn B, Denk W, Bruno RM (2008) In vivo two-photon voltage-sensitive dye imaging reveals top-down control of cortical layers 1 and 2 during wakefulness. Proc Natl Acad Sci U S A 105:7588-7593.

Li CX, Waters RS (1991) Organization of the mouse motor cortex studied by retrograde tracing and intracortical microstimulation (ICMS) mapping. Can J Neurol Sci 18:28-38.

Mitchison G (1992) Axonal trees and cortical architecture. Trends Neurosci 15:122-126.

Moritz CT, Perlmutter SI, Fetz EE (2008) Direct control of paralysed muscles by cortical neurons. Nature 456:639-642.

Nimmerjahn A, Kirchhoff F, Kerr JN, Helmchen F (2004) Sulforhodamine 101 as a specific marker of astroglia in the neocortex in vivo. Nat Methods $1: 31-37$.

Ohki K, Chung S, Ch'ng YH, Kara P, Reid RC (2005) Functional imaging with cellular resolution reveals precise micro-architecture in visual cortex. Nature 433:597-603.

Ozden I, Lee HM, Sullivan MR, Wang SS (2008) Identification and clustering of event patterns from in vivo multiphoton optical recordings of neuronal ensembles. J Neurophysiol 100:495-503.

Poulet JF, Petersen CC (2008) Internal brain state regulates membrane potential synchrony in barrel cortex of behaving mice. Nature 454:881-885.

Pronichev IV, Lenkov DN (1998) Functional mapping of the motor cortex of the white mouse by a microstimulation method. Neurosci Behav Physiol 28:80-85.

Ramanathan D, Conner JM, Tuszynski MH (2006) A form of motor cortical plasticity that correlates with recovery of function after brain injury. Proc Natl Acad Sci U S A 103:11370-11375.

Ramon y Cajal S (1899) Texture of the nervous system of man and the vertebrates. Vienna: Springer.

Sato TR, Gray NW, Mainen ZF, Svoboda K (2007) The functional microarchitecture of the mouse barrel cortex. PLoS Biol 5:e189.

Stosiek C, Garaschuk O, Holthoff K, Konnerth A (2003) In vivo two-photon calcium imaging of neuronal networks. Proc Natl Acad Sci U S A 100:7319-7324.

Weiler N, Wood L, Yu J, Solla SA, Shepherd GM (2008) Top-down laminar organization of the excitatory network in motor cortex. Nat Neurosci 11:360-366. 